\title{
A windfall for US biomedical science
}

\begin{abstract}
In December, the US government approved a $\$ 2$ billion increase in the budget of the US National Institutes of Health (NIH) for the 2016 fiscal year. With that increase comes relief, optimism and the pressure to spend wisely.
\end{abstract}

$\mathbf{F}$ unding for the NIH has been stagnant for the past ten years, with success rates for research project grants (RPGs) at historic lows after falling annually from a 30\% success rate in 2003. In fiscal year 2014, only $18 \%$ of all RPG applications submitted to the NIH were funded. Given that the number of principal investigators applying for those grants has steadily risen since 1980, and that sequestration led to a cut in the budgets of government agencies in 2013, award amounts have been substantially reduced. In 2015, for example, most RPGs awarded by the National Institute on Aging were reduced by $18 \%$ compared to the amount recommended by the relevant study section. As such, an increase of $6 \%$ to the NIH budget in the fiscal year 2016 omnibus bill is welcome news for scientists in the US.

Included in the bill are substantial funding increases for the National Cancer Institute, research into Alzheimer's disease, the Brain Research through Advancing Innovative Neurotechnologies (BRAIN) Initiative and provisions for the Precision Medicine Initiative, which seeks to generate advances that will enable personalized tailoring of therapies to patients. In addition, the bill almost doubles the funding for efforts to prevent and eradicate antibioticresistant bacteria, which pose an increasingly serious threat to human health worldwide.

The boost to the research budget reflects an ongoing push by the current US administration to raise awareness of the importance of science. Recent efforts include securing funding commitments for science, technology, engineering and math (STEM) programs for kids, the establishment of the BRAIN Initiative in 2013, and the release of the National Strategy on Combating Antibiotic-Resistant Bacteria in 2014 and, more recently, of the National Action Plan for Combating Multidrug-Resistant Tuberculosis. The administration also enacted the Food and Drug Administration Safety and Innovation Act in 2012, which helps to accelerate the US Food and Drug Administration (FDA)'s drug-approval process, including of breakthrough therapies. In his State of the Union address on 12 January, President Obama called for an end to malaria and a "moonshot" initiative to cure cancer, to be led by Vice President Biden-lofty goals for the Obama administration's final year in power.

But to achieve these goals, the funding increase must be sustained beyond 2016, and the commitments to new initiatives and strategies need to be honored long beyond the Obama administration's tenure. Cancer will not be cured within 12 months; nor will malaria be eradicated. But given the explosion of technological and research advances in the past ten years alone, the continued growth of investment into research could make these goals attainable in the not-toodistant future.

And despite the funding boost, for some diseases, the budgetary allocation may simply be insufficient to make significant inroads in the near term. In the case of tuberculosis (TB), the monetary commitment needs to be increased to reflect the size of the problem: one-third of the world's population ( 2.4 billion people) is latently infected with TB, and outbreaks of multidrug-resistant TB threaten vulnerable populations. Yet NIH funding for TB was $\$ 279$ million in 2014, compared to more than ten times that amount for HIV, which infects an estimated 37 million people globally. Similarly, raising the funding levels for and the attention given to research in zoonotic diseases-such as those caused by the Ebola virus, dengue virus, the Middle East Respiratory Syndrome coronavirus and newly emerging pathogens such as the Zika virus - and investing in the development of preventive vaccines, cures, surveillance and containment strategies are essential steps to prevent or control future outbreaks. The foresight to commit to long-term funding of prioritized research will be necessary to make the sort of breakthroughs that can have a meaningful impact on human health.

Yet, although it is easy to criticize the budget allocation to the $\mathrm{NIH}$ for not going far enough, it is important to acknowledge that the US government has long been the world's single largest benefactor (in dollars) of both basic and biomedical research. And despite slim increases in the NIH budget over the past decade, science funding in the US still eclipses that in most other countries. By comparison, the German government spent $\sim \$ 19$ billion on science in 2013. Thus, in spite of some prominent US lawmakers who seek to disavow science, the US government's commitment of $\$ 32$ billion to research projects funded by the NIH alone (not including allocations to the National Science Foundation, the FDA, NASA, etc.) is testament to a bipartisan recognition of the importance of investing in scientific research for both US and global benefit. So it is incumbent upon the recipients of those funds to spend them wisely and to recognize the potential global influence of their research decisions. If serious efforts to enhance transparency, reproducibility and the timely reporting of results are combined with studies that build on the technological advances accruing daily, science has the potential to change the landscape of human health as never before. Sustaining the momentum in funding will ensure that these advances have staying power. 